\title{
La preparación de los docentes para la orientación vocacional de adolescentes y jóvenes con síndrome de asperger en condiciones de inclusión
}

\section{The preparetion of the educational directed to the vocational orientation of adolescents and young people with syndrome of asperger under inclution conditions}

\author{
Luis Rodolfo Ramírez Cuellar \\ Universidad Central "Marta Abreu" de Las Villas. Santa Clara. Villa Clara. Cuba \\ ORCID: https://orcid.org/0000-0002-7330-1715 \\ Beatriz Rodríguez Rodríguez \\ Universidad Central "Marta Abreu" de Las Villas. Santa Clara. Villa Clara. Cuba \\ ORCID: https://orcid.org/0000-0002-1318-5425
}

Received 06-12-20 Revised 07-25-20 Accepted 09-13-20 On line 09-30-20

\begin{tabular}{lll} 
*Correspondence & Cite as: \\
\cline { 1 - 1 } Email: eseraggon@gmail.com & & $\begin{array}{l}\text { Ramírez, L., \& Rodríguez, B. (2020). La preparación de los } \\
\text { docentes para la orientación vocacional de adolescentes y }\end{array}$ \\
& jóvenes con síndrome de asperger en condiciones de inclusión. \\
Propósitos y Representaciones, 8 (SPE3), e738. Doi: \\
http://dx.doi.org/10.20511/pyr2020.v8nSPE3.738
\end{tabular}

(C) Universidad San Ignacio de Loyola, Vicerrectorado de Investigación, 2020. 


\section{Resumen}

El presente artículo define las concepciones actuales con respecto a la educación inclusiva, donde se expresan diferentes puntos de vistas al respecto, al inicio se plasma lo que plantea la Organización de las Naciones Unidas para la Educación, la Ciencia y la Cultura (UNESCO) con respecto al tema, luego se enuncian los conceptos expresados por varios autores, de esta manera se puntualiza en que se hace necesario elevar la calidad de la respuesta educativa que requieren los escolares con necesidades educativas especiales en todos los contextos educativos y se enfatiza en aquellos con Trastornos del Espectro Autista, donde se dan sus características a partir de lo que expresa el DSM - V. Seguidamente se hace alusión a la necesidad de preparación de los docentes, de su rol en función de la orientación vocacional de adolescentes y jóvenes con Síndrome de Asperger, se define la misma y se dan criterios de diferentes autores.

Palabras clave: Educación Inclusiva; Preparación; Orientación Vocacional; Asperger.

\section{Summary}

The present article defines the current conceptions with regard to the inclusive education, where different points of views are expressed in this respect, to the beginning that is captured that outlines the Organization of the United Nations for the Education, the Science and the Culture (UNESCO) with regard to the topic, then the concepts are enunciated expressed by several authors, this way it is remarked in that it becomes necessary the quality of the educational answer that the scholars require with special educational necessities in all the educational contexts to rise and it is emphasized in those with Dysfunctions of the Autistic Spectrum, where their characteristics are given starting from what the DSM expresses - V. Subsequently allusion is made to the necessity of preparation of the educational ones, of its list in function of the vocational orientation of adolescents and young with Syndrome of Asperger, he/she is defined the same one and different authors' approaches are given.

Keywords: Inclusive Education; Preparation; Vocational Orientation; Asperger.

\section{Introducción}

En las últimas décadas la Educación Especial a escala internacional ha transitado por un proceso de acelerados y profundos cambios que la han llevado a avanzar desde posiciones paralelas, segregadas en los sistemas educativos hasta las concepciones actuales de plena integración. La complejidad de las relaciones entre educación y sociedad, la marcada necesidad de comprender lo educativo desde la globalidad social, así como las alternativas de atención educativa a lo diverso desde lo común y viceversa, han aportado interrogantes y respuestas que constituyen cruciales temáticas para el debate científico en el ámbito de la Educación Especial.

En el contexto educativo cubano se trabaja para garantizar los plenos derechos y el acceso, sin barreras, a todos los ámbitos sociales de las personas con necesidades educativas especiales, asumiendo una concepción de educación inclusiva, respecto a la cual existen variadas definiciones:

Se considera de utilidad iniciar el análisis por las consideraciones expresadas por la Organización de las Naciones Unidas para la Educación, la Ciencia y la Cultura (UNESCO) sobre este tema, al respecto se plantea: 
(...) puede ser concebida como un proceso que permite abordar y responder a la diversidad de las necesidades de todos los educandos a través de una mayor participación en el aprendizaje, las actividades culturales y comunitarias y reducir la exclusión dentro y fuera del sistema educativo. Lo anterior implica cambios y modificaciones de contenidos, enfoques, estructuras y estrategias basados en una visión común que abarca a todos los niños en edad escolar y la convicción de que es responsabilidad del sistema educativo regular para educar a todos los niños y niñas. El objetivo de la inclusión es brindar respuestas apropiadas al amplio espectro de necesidades de aprendizaje tanto en entornos formales como no formales de la educación; más que un tema marginal que trata sobre cómo integrar a ciertos estudiantes a la enseñanza convencional, representa un enfoque que examina cómo transformar los sistemas educativos y otros entornos de aprendizaje, con el fin de responder a la diversidad de los estudiantes. El propósito de la educación inclusiva es permitir que los maestros y estudiantes se sientan cómodos ante la diversidad y la perciban no como un problema, sino como un desafío y una oportunidad para enriquecer el entorno de aprendizaje. (UNESCO, 2008, p.8).

Muchos autores reconocen a la educación inclusiva como un tema de derecho de todo el alumnado a recibir una respuesta educativa de calidad. $\mathrm{Al}$ respecto se plantea lo siguiente:

Se aspira hacer efectivo para todas las personas el derecho a una educación de calidad, que es la base de una sociedad más justa e igualitaria. La educación es un bien común específicamente humano que surge de la necesidad de desarrollarse como tal, por ello todas las personas sin excepción tienen derecho a ella (Blanco, 2006, p.7).

En esta misma dirección se plantea que la educación inclusiva "es un derecho positivo que, por ello, obliga a las autoridades a crear las condiciones para su disfrute efectivo, removiendo en su caso, las circunstancias u obstáculos que impidan su ejercicio, pues de lo contrario estaríamos ante situaciones de discriminación" (Echeita y Ainscow, 2011, p.31).

Resulta importante señalar que, a partir de la década del 90 en el siglo XX, este concepto emerge para sustituir al de integración, y en el ámbito educativo comienza a promoverse como un complejo proceso relacionado con la necesidad de lograr el cambio y la transformación de las instituciones educativas. Al respecto Booth, Ainscow, Black-Hawkins, Vaughan \& Shaw (2002) declaran que la educación inclusiva:

Se entiende como un proceso de desarrollo que no tiene fin, ya que siempre pueden surgir nuevas barreras que limiten el aprendizaje y la participación, o que excluyan y discriminen de diferentes maneras a los estudiantes. No cabe duda de que la respuesta a la diversidad del alumnado es un proceso que no solo favorece el desarrollo de éste, sino también el de los docentes, las familias y los centros educativos mismos (Shaw (2002, p.7).

Sobre la misma idea de ubicar la esencia de la inclusión educativa dentro de un complejo proceso, versan los criterios de Ramírez (2010), quien señala que se trata de "un conjunto de procesos orientados a aumentar la participación de los estudiantes y las comunidades de las escuelas" (p.158). 
Por otra parte, se reconoce como un proceso que permite abordar y responder a la diversidad de las necesidades de todos los educandos a través de una mayor participación en el aprendizaje, las actividades culturales y comunitarias y reducir la exclusión dentro y fuera del sistema educativo, (UNESCO, 2008, p.8) donde su "objetivo es brindar respuestas apropiadas al amplio espectro de necesidades de aprendizaje tanto en entornos formales como no formales de la educación"... y su "propósito es permitir que los maestros y estudiantes se sientan cómodos ante la diversidad y la perciban no como un problema, sino como un desafío y una oportunidad para enriquecer las formas de enseñar y aprender" (ídem, p.23).

Teniendo en cuenta los aspectos antes señalados por los diferentes autores, en su mayoría coinciden en expresar que la educación inclusiva es un proceso y que además responde a la diversidad de escolares, los autores de la investigación se afilian a los postulados planteado por la UNESCO, por su alcance y dimensión, donde se tiene en cuenta no solo el contexto institucional, sino el familiar y comunitario, aspectos señalados en el presente artículo.

Considerando los elementos anteriores se hace necesario elevar la calidad de la respuesta educativa, que requieren los escolares con necesidades educativas especiales en todos los contextos educativos, adquiriendo particular trascendencia la atención educativa a los niños, adolescentes y jóvenes con Trastornos del Espectro Autista, cuyas principales características se centran en el deterioro persistente de la comunicación social recíproca y la interacción social (Criterio A), y los patrones de conducta, intereses o actividades restrictivos y repetitivos (Criterio B). Estos síntomas están presentes desde la primera infancia y limitan o impiden el funcionamiento cotidiano (Criterios C y D). La etapa en que el deterioro funcional llega a ser obvio variará según las características del individuo y su entorno. (Manual Estadístico y Diagnóstico de Trastornos Mentales (DSM- V).

Las características diagnósticas centrales son evidentes en el período de desarrollo, pero la intervención, la compensación y las ayudas actuales pueden enmascarar las dificultades en, al menos, algunos contextos. Las manifestaciones del trastorno también varían mucho según la gravedad de la afección autista, el nivel del desarrollo y la edad cronológica; de ahí el término espectro. El Trastorno del Espectro Autista incluye trastornos previamente llamados autismo de la primera infancia, autismo infantil, autismo de Kanner, autismo de alto funcionamiento, autismo atípico, trastorno generalizado del desarrollo no especificado, trastorno desintegrativo de la infancia y trastorno de Asperger. (Manual Estadístico y Diagnóstico de Trastornos Mentales (DSM- V).

Esta alteración puede manifestarse desde los primeros años de vida del niño y su aparición dificulta notablemente su incorporación a la sociedad. A pesar de las investigaciones científicas realizadas desde mediados del pasado siglo (Kanner, 1943; Asperger ,1944; L. Wing, 1982; Riviere, 1990; Frith, 1991, Gray C, y Castellanos, 1995. y Tamarit J, 1998, entre otros.), el autismo sigue siendo una interrogante no completamente resuelta, un problema sin suficientes respuestas, un desafío para las ciencias médicas, pedagógicas, psicológicas, neurológicas, genéticas, bioquímicas, entre otras.

La problemática relacionada con la atención a los Trastornos del Espectro Autista ha sido un tema tratado desde la ciencia en Cuba desde diferentes puntos de vistas desde el campo de la psiquiatría: Martínez. C, (1998); Rodríguez. O; Velásquez. J, (2001); Ravelo. V (2002), entre otros; en el ámbito pedagógico se destacan los estudios realizados por Massani Enríquez J.F 
(2009), Escalona. E. (2010) la cual ofrece un sistema de evaluación psicopedagógica para los niños con Autismo, Demóstenes. Y (2010), trabaja la socialización en los niños con Autismo; Campos. I, (2012) abordó la temática de la educación familiar en la escuela para niños con Autismo; Orozco Delgado, M (2012). Trabajó en una concepción pedagógica de atención integral a los niños y adolescentes con diagnóstico de Autismo.

En la provincia de Villa Clara, Cuba, se desarrollaron las tesis de Díaz Sifontes, H (2011), Torres Mollineda, G. L (2013) de especialización en Psicopedagogía, quienes dirigieron su investigación a la preparación de los especialistas de los Centros de Diagnóstico y Orientación (CDO) y docentes para trabajar con niños que presentan conducta autista. Los elementos anteriores demuestran que se han realizado estudios sobre los Trastornos del Espectro Autista, pero es necesario destacar que estos no se dirigen a la Orientación Vocacional de adolescentes y jóvenes portadores de Síndrome de Asperger incluidos en la Educación General.

Sin lugar a dudas los resultados aportados por estos investigadores sirven de referente al estudio que se realiza para favorecer la preparación de los docentes en función de la Orientación Vocacional en los casos de Síndrome de Asperger. Las personas con Síndrome de Asperger a diferencia del Autismo clásico no presentan dificultades en la resolución de las tareas que evalúan capacidades mentales, sin embargo en las situaciones del día a día, en su ambiente natural, es donde muestran serios problemas, especialmente en las relaciones sociales, en interpretar y leer las intenciones de los demás, así como en sus conductas o procesos de pensamiento que suelen ser inflexibles, rígidos, repetitivos y perseverantes, de ahí la importancia de orientarlos en el orden vocacional.

\section{Preparación de los docentes}

Los profesionales que tratan con escolares tan diversos necesitan adquirir cada vez más conocimientos en el área, así como las habilidades necesarias, es decir mantenerse actualizados y bien preparados lo que se refleja en el siguiente principio basado en que todos los especialistas tienen la responsabilidad de mantener una elevada competencia profesional. Si labora con personas que presentan defectos discapacitantes, este principio cobra un valor importantísimo, ya que las decisiones que puede tomar un profesional en esta área ponen en juego el futuro del sujeto. (Castro ,2002).

Desde ese punto de vista se requiere que, desde las etapas iniciales de preparación del docente, su motivación profesional contribuya irrevocablemente a sus reflexiones, por lo que es necesario asumir ese reto para cumplir su misión social.

El proceso de atención a escolares con necesidades educativas especiales, como todo tipo de actividad de aprendizaje, tiene bases organizativas generales que se ajustan y no se contraponen a las metodologías especificas preestablecidas para cada contenido del programa, sin embargo, el análisis de la instrumentación de este proceso en la práctica induce a señalar que hay que preparar bien a los docentes para que realicen las acciones pensando en las potencialidades de estos para lograr una verdadera integración de todas las actividades. 
Se deberá capacitar más a los docentes, para que eleven su preparación y puedan promover el progreso de los escolares, adquieran los conocimientos que les permitan potenciar el desarrollo. Es lograr que los escolares llamados diferentes tengan un espacio para que muestren sus conocimientos y habilidades.

Estas ideas son válidas para reflexionar y promover ayudas metodológicas que den respuesta a la atención a esa diversidad. Dentro de los temas metodológicos a desarrollar pueden encontrarse: estudio del diagnóstico de todos, para descubrir a tiempo las fortalezas, debilidades y necesidades, diseño de estrategias educativas integrales, el trabajo preventivo, adaptaciones curriculares y calidad de vida.

Lo anterior es premisa esencial en la preparación del docente, el cual debe reconocer que es extremadamente indebido ofrecer igual tratamiento a escolares con niveles de desarrollo y necesidades muy distintas. Sobre la base de este elemento, conocimientos y habilidades él deberá lograr el desarrollo máximo de capacidades de acuerdo con las posibilidades de cada uno.

Se considera oportuno puntualizar algunas EXIGENCIAS para mantener una postura optimista, potenciadora y una ética profesional de atención a la diversidad, lo cual hace que el docente se sienta preparado.

- No olvidar la unidad que existe entre lo afectivo/cognitivo en el desarrollo de la personalidad: lo anterior presupone realizar un diagnóstico psicopedagógico actualizado donde se indague en los motivos, necesidades, intereses, deseos, con la misma profundidad que cuando se estudia sus capacidades intelectuales.

- Brindar atención a todos a partir de las diferencias individuales utilizando formas, métodos, procedimientos, técnicas y medios, de manera creativa.

- Estudio sistemático de la bibliografía relacionada con la Educación y Diversidad: significa mantenerse actualizado e instrumentar en el quehacer pedagógico los conocimientos adquiridos, sobre la base de la autosuperación.

- El docente debe ser ejemplo de educador, altruista, sensible, con la concepción optimista de educar y enseñar a cada cual según sus necesidades: significa que debe lograr organizar el proceso de enseñanza aprendizaje para dar respuesta al fin de la sociedad respecto a la atención a la diversidad .Su ejemplo es concluyente para el logro de una acción educativa eficaz y perdurable por el impacto que tiene en la formación de la nueva generación y así se convertirá en el fundamento de su actividad transformadora.

Ahora bien ¿qué se entiende por orientación vocacional?

La orientación vocacional puede ser entendida como un proceso que ayuda a la elección de la profesión (Vidal y Fernández, 2009). Se trata de la actividad encargada de cubrir las necesidades que preparan dicha elección, y que van desde favorecer el conocimiento de los intereses propios hasta la evaluación de las posibilidades reales de acceso a su ejercicio, su evaluación y su seguimiento.

Es también un conjunto de conocimientos y prácticas que buscan asegurar que los adultos jóvenes se formen en actividades profesionales que se correspondan con su interés personal, y al mismo tiempo, aseguren un rendimiento eficiente en sus futuros entornos laborales. 
Dicho proceso no sólo tiene el objetivo de incorporar a un adulto joven a la fuerza laboral profesional y guiarlo durante su ejercicio, sino que requiere además reconocer el interés de la persona y facilitar su aprendizaje sobre el entorno laboral.

De acuerdo con Molina (2001), la orientación vocacional en la Educación Básica es un proceso dirigido al conocimiento de diversos aspectos personales: capacidades, gustos, intereses, motivaciones personales, en función del contexto familiar y la situación general del medio donde se está inserto, para poder decidir acerca del propio futuro.

Los cambios que ha experimentado el concepto de orientación son consecuencia de la evolución de los factores históricos y sociales. Así, en sus orígenes y hasta 1925, el concepto es consecuencia de necesidades socioeconómicas: ajustar las características y capacidades de cada persona a los requerimientos de una profesión, con el objetivo de alcanzar el máximo rendimiento en el trabajo (Vidal, 2001; González \& Omaira, 2003).

Un aspecto a señalar, en este sentido, es lo planteado por el Dr. John D. Krumboltz de la Universidad de Stanford en la conferencia inaugural del Congreso de la Association for Educational and Vocational Guidance (IAEVG), celebrada en Suiza, donde indica que para estos tiempos es necesario hacer nuevas consideraciones relacionadas con los objetivos de la Orientación Vocacional, además de tomar en cuenta los eventos fortuitos, «casualidades» u oportunidades que se dan alrededor de las personas (De León \& Rodríguez, 2008) .

En las definiciones anteriores se recrea la práctica de la orientación en lo vocacional, donde el orientador, que bien puede ser el docente, ha recibido un entrenamiento y preparación que le permite ofrecer asesoría y asistencia a los alumnos y les ayuda a tomar decisiones para progresar con éxito en su vida estudiantil y profesional, en tal sentido los autores se afilian al concepto dado por Vidal y Fernández (2009).

\section{Rol del docente en la orientación vocacional}

El docente juega un papel muy importante en el desarrollo de la orientación vocacional, ya que es quien más tiempo pasa con el alumno en el aula, y es quien de cierta forma sirve como modelo frente al estudiante en su ejercicio como docente.

El objetivo de estas intervenciones es el esclarecimiento de la problemática vocacional, dotándoles de los elementos necesarios para posibilitar la mejor situación de elección para cada alumno. Se puede concretar tanto de forma individual como grupal, ya que supone actividades ligadas tanto a la exploración personal como al análisis de la realidad a través de información sobre la oferta académica y las particularidades del mercado laboral, así como teniendo en cuenta en cada momento sus capacidades e intereses específicos, imprescindibles para el éxito laboral de una persona con síndrome de Asperger.

Se pueden ofrecer Talleres grupales de motivación para el empleo, desde las que se pueden trabajar las siguientes temáticas:

- Habilidades Socio-laborales

- Motivación para el empleo 
- Técnicas de Búsqueda Activa de empleo: Carta de Presentación, Herramientas para la búsqueda activa de empleo, etc.

- Entrenamiento, entrevistas de trabajo.

\section{Participantes}

De una población de 47 escolares con diagnóstico de Trastorno del Espectro Autista incluidos en los diferentes niveles educativos, se escogió una muestra de 13 adolescentes y jóvenes con Síndrome de Asperger, lo que se muestra en la siguiente tabla:

\begin{tabular}{lcccccc}
\hline \multirow{2}{*}{\begin{tabular}{l} 
Nivel educativo \\
\cline { 3 - 7 }
\end{tabular}} & Total & $13-14$ & $14-$ & $16-17$ & $\mathrm{M}$ & $\mathrm{F}$ \\
Secundaria Básica & 5 & 5 & & & 5 & \\
$\begin{array}{l}\text { Educación de } \\
\text { jóvenes y adultos }\end{array}$ & 1 & - & - & 1 & 1 & - \\
$\begin{array}{l}\text { Educación Técnica } \\
\text { y Profesional }\end{array}$ & 7 & - & - & 7 & 6 & 1 \\
Total & 13 & 5 & - & 8 & 12 & 1 \\
\hline
\end{tabular}

En cuanto a los docentes

\begin{tabular}{lccccccccccc}
\hline & & \multicolumn{1}{c}{ Docentes } & \multicolumn{3}{c}{ Sexo } \\
\cline { 3 - 7 } $\begin{array}{l}\text { Nivel } \\
\text { educativo }\end{array}$ & Total & $\begin{array}{c}\text { Guía } \\
\text { de } \\
\text { grupo }\end{array}$ & $\begin{array}{c}\text { Profesor } \\
\text { de } \\
\text { asignatura }\end{array}$ & Directivo & $5-10$ & $10-15$ & $15-20$ & $\begin{array}{c}20 \text { y } \\
\text { más }\end{array}$ & M & F \\
S/ B & 16 & 6 & 7 & 3 & 5 & 6 & 2 & 3 & 7 & 9 \\
E.J.A & 7 & 3 & 2 & 2 & - & - & 3 & 4 & 2 & 5 \\
E.T.P & 13 & 5 & 5 & 3 & 2 & 5 & 1 & 5 & 8 & 5 \\
Total & 36 & 14 & 14 & 8 & 7 & 11 & 6 & 15 & 17 & 19 \\
\hline
\end{tabular}

Leyenda

S/B - Secundaria Básica.

E.J.A - Educación de Jóvenes y Adultos.

E.T.P - Educación Técnica Profesional.

\section{Métodos}

\section{Del nivel teórico}


- Histórico-lógico: se empleó para analizar, valorar y tomar posiciones en relación con la evolución histórica de la atención educativa a los escolares con Autismo.

- Análisis documental: permitió analizar las políticas que tienen relación con la atención educativa a los escolares con Trastorno de Espectro Autista y sus particularidades.

- Análisis y síntesis: se utilizaron en todos los momentos de la investigación al igual que la inducción-deducción a los efectos de lograr las abstracciones y generalizaciones necesarias, ambos proporcionaron la posibilidad de realizar valoraciones acerca de la información recogida en fuentes bibliográficas y en los instrumentos aplicados para la constatación empírica del problema.

- La modelación: para la determinación de la estructura de la estrategia a proponer.

- Enfoque de sistema: se utilizó en función de establecer las interrelaciones y monitorear los diferentes componentes que conformarán la estrategia.

\section{Del nivel empírico}

- Observación: en actividades que desarrollan los docentes.

- Entrevista: a directores de las escuelas, y a los especialistas de los Centros de Diagnóstico y Orientación con el objetivo de constatar el estado de la atención de los adolescentes y jóvenes con Síndrome de Asperger, sobre todo en lo concerniente a la orientación vocacional.

- Encuesta: a los docentes para constatar la atención que le brindan a los adolescentes y jóvenes en cuanto a su orientación vocacional.

- Criterio de especialistas: Se empleó para obtener valoraciones acerca de la factibilidad y viabilidad de la estrategia propuesta y hacer recomendaciones con un máximo de competencia.

Métodos matemáticos y estadísticos: Para el procesamiento de la información se utilizaron los métodos y procedimientos de la estadística descriptiva entre ellos la media $\overline{\mathrm{X}}$ y el cálculo porcentual.

\section{Resultados}

Los resultados de las prácticas pedagógicas de los docentes cubanos, la experiencia profesional de los autores de este trabajo, relacionado con el estudio diagnóstico de los niños, adolescentes y jóvenes con Trastornos del Espectro Autista en la provincia, los resultados de los métodos aplicados, ha permitido plantear empíricamente la identificación de las principales dificultades en la atención educativa de los escolares con Autismo en nuestro contexto.

- Se aprecian:

- Limitaciones en el proceso de diagnóstico clínico y la investigación psicopedagógica del Autismo.

- Deficiencias en el diseño de la respuesta educativa, teniendo en cuenta las particularidades de los diferentes contextos educativos, en que se asegura la atención.

- Insuficiencias de carácter organizativo, docente y científico - metodológico para la atención educativa.

- Insuficiencias en lo concerniente a la orientación vocacional en función de su preparación para la vida adulta e independiente.

- Insatisfacciones en la implicación de la familia y otros agentes educativos en lo relativo al proceso de atención educativa. 
Lo anterior revela la contradicción entre la necesidad de perfeccionar la atención educativa de los escolares con Trastorno del Espectro Autista y la falta de una concepción pedagógica científicamente fundamentada para realizar este proceso, lo que condujo a la necesidad de elaborar una estrategia dirigida a la preparación de los docentes en función de la orientación vocacional de los adolescentes y jóvenes con Síndrome de Asperger que se encuentran incluidos en las escuelas de la educación general.

\section{Impactos de la investigación}

Los impactos se aprecian fundamentalmente en el orden social, en la ética del profesional, ya que contribuyen a la inserción socio laboral activa de los adolescentes y jóvenes con Síndrome de Asperger, además de la educación de la sociedad en la aceptación a este tipo de persona.

Otras de las prioridades a las que tributan los resultados están referidas al desarrollo humano, equidad y justicia social. Da cumplimiento a una de las prioridades del sector educacional en cuanto a la orientación vocacional, la continuidad de estudio, así como la relacionada al trabajo preventivo.

Se va a preparar a los docentes que tienen adolescentes y jóvenes en sus aulas con Síndrome de Asperger con enfoque desde la educación inclusiva. Se realiza una sistematización de los estudios recientes en cuanto a los trastornos del espectro autista, sobre todo lo referido a la Orientación Vocacional en adolescentes y jóvenes con diagnóstico de Síndrome de Asperger, incluidos en la Educación General.

Responde al proyecto "La Pedagogía Especial como sustento para la inclusión socioeducativa de los escolares con Necesidades Educativas Especiales (NEE)", el cual está asociado al programa que se desarrolla en el departamento de Educación Especial de la Facultad de Educación Infantil de la Universidad Marta Abreu de Las Villas. Cuba.

\section{Conclusiones}

Contar con un alumno Asperger dentro de un aula regular, requiere aceptación y respeto, además de un ambiente real que le permita desarrollarse como persona en forma integral, por lo que es necesaria la participación de todos los elementos del ámbito educativo. Es necesario considerar que cada alumno con Síndrome de Asperger es único por lo que requiere que los docentes se informen sobre la condición y diseñen intervenciones de acuerdo a las necesidades de sus alumnos.

El proceso de inclusión debe involucrar a todos los actores del centro educativo, considerando como parte fundamental el apoyo de la familia y los padres.

La orientación vocacional desde el espacio educativo se plantea como un proceso continuo, debe ser promovida desde la gestión de la institución educativa, además de considerar en los escolares con Síndrome de Asperger el trabajo permanente en las áreas curriculares y desde la tutoría.

\section{Referencias}

Alonso, J. (2009). Autismo y Síndrome de Asperger. AMARU. Ediciones Salamanca. 
Álvarez, C. (1999). El impacto de la Teoría de Vigotski en la Educación Especial en Cuba. Informe presentado al examen de Problemas sociales de la ciencia. ICCP. MINED.

Asociación Americana de Psiquiatría. (2014). Manual Estadístico y Diagnóstico de Trastornos Mentales (DSM- V).

Blanco, G.R. (2006). La equidad y la inclusión social: uno de los desafíos de la educación. REICE - Revista Electrónica Iberoamericana sobre Calidad, Eficacia y Cambio en Educación, 4(3), 1-15.

Booth, T. y Ainscow, M. (2002). Índice de inclusión. Desarrollando el aprendizaje y la participación en las escuelas. Santiago de Chile: ORLEAC/UNESCO.

Booth, T., Ainscow, M., Black-Hawkins, K., Vaughan, M., \& Shaw, L. (2002). Centre for Studies on Inclusive Education (CSIE), Bristol UK 2000.

Booth, T., y Ainscow, M. (2015). Guía para la Educación Inclusiva: Desarrollando el aprendizaje y la participación en los centros escolares (3ra edición).

Borges S. (2007). Atención integral a las personas con necesidades educativas especiales en Cuba. Curso \#14. Congreso Internacional Pedagogía 2007. La Habana: Ministerio de Educación.

Campos. I. (2012). La educación familiar en la escuela para niños con Autismo. [Tesis en opción del título académico de doctor en Ciencias Pedagógicas]. La Habana.

Castro, P.L. (2008). El maestro y la familia del niño con discapacidad. La Habana: Editorial Pueblo y Educación.

De León, T y Rodríguez, R (2008). El efecto de la orientación vocacional en la elección de carrera. Revista Mexicana de Orientación Educativa, 5(13), 12-20.

Declaración de Salamanca. (1994). Marco de acción para las Necesidades Educativas Especiales, España.

Demosthene, Y. (2003). Alternativa para el desarrollo de la conducta social. [Tesis en opción al Título Académico de Máster en Educación Especial]. La Habana: Centro de Referencia Latinoamericana para la Educación Especial.

Echeita, G., \& Ainscow, M. (2011). La educación inclusiva como derecho. Marco de referencia y pautas de acción para el desarrollo de una revolución pendiente. II Congreso Iberoamericano de Síndrome de Down.

Educación especial. (2008). Inclusión educativa. V Jornadas. Oficina Regional para América Latina y el Caribe. Cartagena de Indias.

Frontera, M. (2016). Alumnos con Síndrome de Asperger. Necesidad y respuesta educativa, Madrid.

Massani, J. (2009). Metodología para el diagnóstico psicopedagógico de niños y niñas con autismo. Un estudio de caso en la provincia de Cienfuegos. [Tesis en opción al Grado Científico de Doctor en Ciencias Pedagógica]. Cienfuegos, Cuba. 
Organización de las Naciones Unidas para la Educación, la Ciencia y la Cultura (Unesco). (1994). The Salamanca Statement and framework for action on Special Needs Education. Paris.

Organización de las Naciones Unidas para la Educación, la Ciencia y la Cultura (Unesco). (1994). Declaración de Salamanca y Marco de Acción para las necesidades educativas especiales. Salamanca.

Organización de las Naciones Unidas para la Educación, la Ciencia y la Cultura (Unesco). (2008). Educación inclusiva: camino hacia el futuro. Unesco.

Organización de las Naciones Unidas para la Educación, la Ciencia y la Cultura (Unesco). (2019). Inclusión en la educación. Recuperado de: https://es.unesco.org/themes/inclusioneducacion

Orozco, M (2012). Concepción pedagógica de atención integral a los niños y adolescentes con diagnóstico de Autismo. [Tesis en opción al título académico de doctor]. La Habana.

Ramírez, C, S. (2010). Educación inclusiva y la estimulación temprana. ARJÉ Revista de Postgrado FACE-UC., 153-170.

Sosa, L. (2006). Proyecto Educativo "Dora Alonso" para la atención integral a los niños con autismo. [Tesis en opción del Título Académico de Máster en Educación Especial]. Centro de Referencia Latinoamericano para la Educación Especial. La Habana.

Torres, G.L. (2013). La preparación de los docentes para trabajar con niños que presentan conducta autista. [Tesis de especialización en Psicopedagogía], Villa Clara. Cuba.

Vidal, M., \& Fernández, B. (2009). Orientación vocacional. Revista Educación Media Superior $23(2), 1-11$. 